\title{
Nurses' Resuscitation Performance: Study on the Effectiveness of Training and Support at a Teaching Hospital in KSA
}

\author{
Ouseph, $\mathbf{B}^{1, *}$, Mohidin, $\mathrm{S}^{2}$, Tabsh, $\mathbf{L}^{3}$, Al-Hebshi, $\mathrm{A}^{4}$ \\ ${ }^{1}$ Nursing Education \& Development Unit, King Abdulaziz University Hospital, Saudi Arabia \\ ${ }^{2}$ Nursing Clinical Practice \& Research Unit, King Abdulaziz University Hospital, Saudi Arabia \\ ${ }^{3}$ Nursing Administration, King Abdulaziz University Hospital, Saudi Arabia \\ ${ }^{4}$ Hospital Administration, King Abdulaziz University Hospital, Saudi Arabia
}

Copyright $\mathrm{O} 2015$ by authors, all rights reserved. Authors agree that this article remains permanently open access under the terms of the Creative Commons Attribution License 4.0 International License

\begin{abstract}
Introduction: This paper discussed nurses' resuscitation trainings and their performance in a teaching hospital based on AHA guideline recommendations. The study explored the current standard practices and investigates the nurses' views on the effectiveness of the training, quality of equipment, familiarity and readiness of nurses in performing resuscitation. Methodology: A cross-sectional descriptive study conducted at teaching hospitals which mandate all nurses must be certified with the BLS and attends two code blue drill sessions per year. A self-administered questionnaire examined the support and guidance provided, the availability of resuscitation equipment and their quality/familiarity, the quality of training received in performing the resuscitation actions and finally explored the opinion in improving resuscitation. Results: The study revealed that the organization has established system to prepare the nurses adequately. Based on the 192 nurses, the total mean 2.63 supported that nurses are adequately trained with resuscitation knowledge and skills, $95 \%(n=171)$ of nurses were confident in announcing the code blue procedure, $93.3 \% \quad(n=169)$ received appropriate training and $90 \%(\mathrm{n}=162)$ found training instructors utilized standard method of simulation training which $90 \%(\mathrm{n}=161)$ shared a real-life clinical scenarios are used in the training whilst $90.5 \%(n=162)$ stated SBAR is utilized as mode of communication and $66.6 \%(n=120)$ practiced post-procedure resuscitation debriefing. However, concerns were raised in three areas: availability of AEDs, cable and pad accessibility and permission to perform defibrillation procedures. In conclusion, study established nurses were well-prepared, trained and supported for resuscitation care.
\end{abstract}

Keywords Resuscitation, Nursing Care, CPR

\section{Introduction}

It is important to provide effective cardiopulmonary resuscitation care to arrested patients to improve mortality and morbidity rates [1]. Per the AHA recommendations, every minute that CPR is delayed decreases survival rates [2]. For the purpose of optimal performance, it is ideal to provide standardized equipment and regular trainings based on international guidelines [3]. Cardiopulmonary resuscitation has been practiced for many years, and many studies have shown that when resuscitation knowledge and skills are applied correctly, it can maintain perfusion until the return of spontaneous circulation [4]

Numerous studies have established that all healthcare professionals involved in direct patient care have to receive compulsory training and resuscitation equipment should be always readily available for resuscitation care [5]. A previous study demonstrated that cardiac arrest performances improved when all nurses were certified in the relevant life support training courses [2]. Clinical area nurses also need to be trained in the automated external defibrillation procedure and should have access to such devices, as it will prevent delays in basic life support care in resuscitation. Early defibrillation in cardiac arrest with VF should take priority in resuscitation, and the probability of survival progressively decreases when defibrillation is delayed for more than 10 minutes. A study suggested that such skills as cannulation, drug administration, intubation, and ventilation could subsequently be developed in order to enhance cardiac arrest care [7].

It is suggested that whenever CPR is initiated, it should be performed within the accepted clinical guidelines [11]. Studies recommend that the use of international standards in cardiopulmonary resuscitation care and effective training and assessment will result in better basic life support knowledge and skills during actual procedures [14]. As per 
the AHA's recommendations, nurses in healthcare settings should be certified in Basic Life Support, and critical care nurses need ACLS status along with Basic. The nurses should be re-certified every two years [2].

Resuscitation skills in cardiopulmonary resuscitation can be gained through mannequin training and assessment can be performed with an observational check list. Frequent refresher trainings are needed to maintain such skills [8]. Resuscitation training methods should be evaluated frequently in order to improve the resuscitation practice [9].

Recent literature identified that resuscitation skills and knowledge can decline over time even after effective training and retention can be achieved by regular training every 6 months [10]. Across the world, learners are failing to reach the required standards in resuscitation care and are unable to achieve the chain of survival for saving lives [12].

Standardized teaching methods may result in retention of resuscitation skills among nurses and the use of standardized teaching styles may facilitate the retention of resuscitation skills. Standardized equipment and its familiarity are preferred in the clinical areas [13].

The AHA 2010 guidelines are available now and emphasize that any attempt at CPR is better than no attempt at all. In order to remove any barriers, such as wrong techniques and fear of exposure to infectious diseases, the guidelines recommend only applying compression techniques, and the responder should not wait for any equipment in the initial steps of resuscitation. The requirements are to perform the action with chest compressions, which was re-emphasized in the guidelines. These practices can achieve the objectives of resuscitation care [2].

Nurses in clinical settings desire to learn CPR, as they are aware that early cardiopulmonary resuscitation can save lives. These nurses have the ability to improve the survival rate of a patient, and they are also committed to provide such care [15] Most of the time nurses are the first responder who witnesses the arrest in the hospital and as a first responder in a cardiac arrest situation, she or he needs to take appropriate actions, such as recognition, shouting for help, initiation of BLS, application of AED and reporting to the code blue team using the SBAR communication method [16]

For the purpose of maintaining resuscitation knowledge and skills, we must pay attention to the training quality, recognizing the need for increasing the monitoring of adherence to recommended guidelines and also identifying areas for improvement, including the quality of clinical resuscitation equipment. [4, 18, $19 \& 20]$.

Therefore, this study will explore the current practices in the studied hospital and examine the effectiveness of the training and support provided to the nurses.

\section{Aims and Objectives of Research}

The study was conducted in two parts: first, studying the standard practice in the studied hospital and, second, exploring the nurses' perspectives toward: support and guidance given to the nurses for resuscitation care resuscitation equipment and its quality level current training standards based on AHA recommendations areas for improvement in the resuscitation practice.

\section{Significance of the Study}

This study may identify the barriers in providing effective resuscitation care to patients. It may assist in motivating resuscitation team members and purchase teams to purchase good quality resuscitation equipment for clinical use. The study can also guide the hospital personnel to keep standardized equipment in the resuscitation trolley in order to avoid confusion of their use. The study may suggest permitting the nurses to perform certain procedures in resuscitation, especially in the use of AED and defibrillation. The need for continuing mandatory trainings and their duration can be recommended based on the study results. This study may support influences on the training quality and recognize the need for increasing the monitoring of adherence to the recommended guidelines.

\section{Methods}

A cross-sectional descriptive study is used to evaluate the organization's current practice and nurses' perspectives on resuscitation care.

This study took place in an 800-bed teaching hospital, known as a tertiary care center. The teaching hospital consists of 24 clinical areas. The hospital has a large number of nurses (1066, based on the Nursing administration December 2014 statistic). The study was open to all categories of nurses, including Head Nurses, Deputy Head Nurses, Clinical Instructors and staff nurses. Inclusion criteria were set to recruit the participants.

Table 1. Selection criteria Inclusive criteria

\begin{tabular}{|c|c|}
\hline Inclusive criteria & Exclusive criteria \\
\hline $\begin{array}{l}\text { Nurses who are } \\
\text { completed BLS } \\
\text { certification course and } \\
\text { holding valid certificate } \\
\text { Attended minimum two } \\
\text { code blue drills per year. } \\
\text { Nurses who are still } \\
\text { actively working in the } \\
\text { clinical areas } \\
\text { Nurses are interested to } \\
\text { participate in the study }\end{array}$ & $\begin{array}{l}\text { Do not attend resuscitation } \\
\text { drill training for the last } \\
\text { one year } \\
\text { Nursing staff who has } \\
\text { medical problems and } \\
\text { concerned conditions } \\
\text { present in the drill } \\
\text { Nursing administration } \\
\text { team }\end{array}$ \\
\hline
\end{tabular}

The sample size was decided using a Rao soft sample size calculator with a total population of 1066, a confidence level of $95 \%$ and a confidence interval of 5 . The sample size required was 283 participants. A larger sample was selected as we assumed the nonresponse rate would be approximately $20 \%$. For the purpose of getting a target sample, the study was conducted for a few consecutive months and the sample distributed after the training and collected within one to two weeks. 
A questionnaire was utilized as the tool for data collection. There are several categories of items included in the questionnaire: support and guidance received from the institution for providing resuscitation care, availability of resuscitation equipment and its quality/familiarity, quality of training received on performing the resuscitation actions and finally their observations or comments on improving resuscitation.

Permission was taken from the Nursing Department of the hospital and the Research Biomedical ethics committee. The participants were given a brief explanation of the purpose of the study. The study was carried out anonymously on a voluntary basis, and confidentiality was assured.

Questionnaires were distributed after the scheduled drill training, and participants were asked to not leave any questions unanswered.

\section{Results}

\section{I: Study on the Current Practices of the Organization}

The organization has a standard practice that all nurses are certified with the BLS with mandatory training drills, either in the unit or in the class room.

\section{i. Resuscitation Training Is Based on the AHA}

The training conducted in the teaching was based on the American Heart Association (AHA) standards. The American Heart Association has helped in developing the concepts of emergency cardiovascular care, and it has five links in the adult chain of survival: immediate recognition, early cardiopulmonary resuscitation, rapid defibrillation, effective advanced life support care and integrated post-cardiac-arrest care. BLS certifications for all nurses are completed within the first two months of the probationary period. An additional Advance Life Support Training(s) appropriate for the area of critical care practice is conducted within the first year of contract.

Therefore, all of the nurses who received the training will be expected to accomplish several actions simultaneously and be involved in the above recommended care per their roles in resuscitation. Through standardized trainings, good quality equipment familiarity through simulated trainings, the nurses will be able to take part in their assigned roles effectively.

\section{ii. Nurses' Roles during Resuscitation}

The scope of nurses' professional practice also allows the freedom to take part in actual resuscitation care after their formal certification and two refresher drill training programs.
Nurses are also responsible for checking resuscitation trolleys on a daily basis and ensuing that the trolleys are readily available in all clinical areas. The nurses regularly confirm all resuscitation items are in good working condition, and the trolley is kept locked for emergency resuscitation care.

\section{iii. Resuscitation Training}

Resuscitation training for the nurses in the teaching hospital is designed to contribute to the management of cardiopulmonary arrest in hospital settings. The training concepts are divided into two main roles for nurses. The primary role is basically to provide basic life support care during resuscitation. The secondary role consists of crash cart awareness, equipment familiarity, skills in handling the equipment, defibrillator safety, accurate documentation and participation in the post-resuscitation care and debriefing. The hospital has adequate resuscitation officers (a total of 4 based on 2014 hospital statistics) as per the UK resuscitation council recommendation; 1 to every 750 staff in training. It is required that all nurses need to attend at least two sessions of code drill (hands-on) exercises every contract year. The drills are for updating the knowledge and skills of nurses in resuscitation. The drills are conducted either in the clinical areas or classrooms. A mock code blue drill is carried out to assess team approach care, which includes promptly recognizing the arrest within 10 seconds, code blue activation, high quality BLS skills, including initiating the chain of survival, crash cart awareness, defibrillator safety, ECC drug preparation and administration, and cardiopulmonary documentation.

However, with all the data stated above, it still has been repeatedly reported by the evaluators that nurses who attended the training have difficulty using their skills in actual resuscitations, and they often forget the learned knowledge and skills rapidly. Studies of CPR evaluations confirmed that skill performance begins to decline within several days of initial training [2]. Hospitals also provide a crash cart in all clinical areas and are always ready with resuscitation equipment; therefore, its use is also included in the training program. Nurses have access to the hospital policies and their primary roles in BLS and secondary roles in ACLS are clearly stated, to remove barriers in resuscitation care. Unfortunately, in spite of all these preparations, nurses are not able to use their knowledge and skills effectively during actual resuscitation procedures. This triggers the researchers' interest in studying nurses' effectiveness based on the quality of Resuscitation equipment, their familiarity with Crash Cart items, their training quality and their readiness for such care. 


\section{$\%$ Certified /Valid attendee}

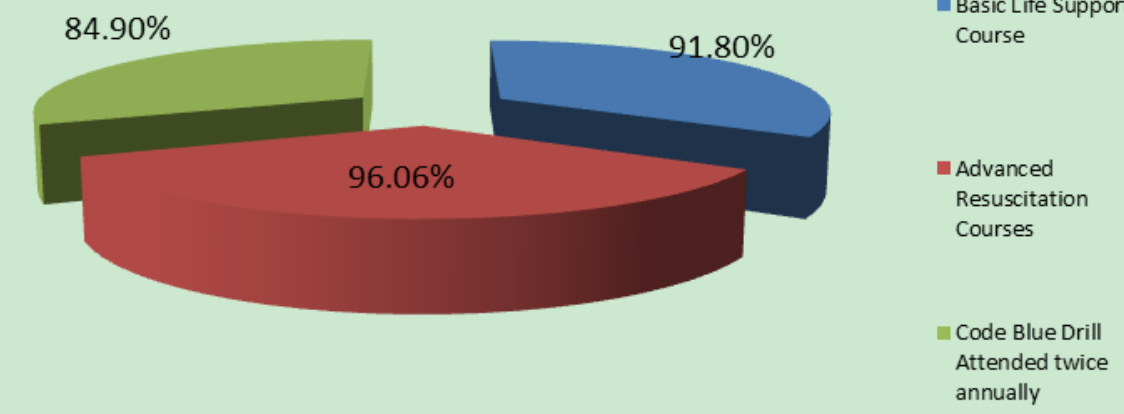

Figure 1. Total number of certified staff with life support courses

\section{Results II: Demographic Analysis}

All completed data were included in the analysis using SPSS Windows Version 16. Although the target sample was 283, only 192 nurses agreed to answer questionnaires and 182 participants returned them with a drop rate of $5.4 \%$. Two questionnaires were incomplete and have been eliminated from the analysis.

\section{Finding 1: Quality of Training: Nurses' View}

Most of the components were appraised between "strongly agree" and "disagree" and the total mean shown of 2.63 in the survey supported that the training is effective (Table 2).

Table 2. Quality of training

\begin{tabular}{|c|c|c|c|}
\hline & $\mathrm{N}$ & Mean & Std. Deviation \\
\hline Quality of training & 180 & 2.63 & .518 \\
\hline
\end{tabular}

In detail, the participants showed strong agreement with four statements in which $95 \% \quad(n=171)$ of nurses demonstrated that they are confident in announcing code blue, $93.8 \%(n=169)$ received appropriate training prior to such care, and $90 \%(\mathrm{n}=162)$ said their training instructors use standardized methods of simulation training for retaining the learned skills. The use of real life clinical scenarios in the training was also explored, and $90 \%(\mathrm{n}=161)$ of the nurses agree with it.

The participants were asked to assess the quality of training they received and $63.7 \%(n=116)$ were extremely satisfied, $33.5 \%(\mathrm{n}=61)$ were somewhat satisfied and $1.6 \%$ $(n=3)$ were not satisfied. Nurses' confidence level in the resuscitation documentation was $88.3 \% \quad(\mathrm{n}=158) .95 \%$ $(n=169)$ approve of the instructors availability and the availabilities of regular training.

\section{Finding 2: Support and Guidance Provided for Resuscitation Care}

This part of the study explored the support and guidance given to the nurses for providing resuscitation care in the teaching hospital. The result reflected that the hospital had established a process to train $97.2 \%(n=175)$ of the nurses with the gold standard BLS certification program during their probationary period and $98.3 \%(n=177)$ nurses were recertified every two years. Additionally, $98.4 \%(\mathrm{n}=177)$ of nurses were professionally responsible for their CPR competency, and $96.6 \% \quad(n=174)$ agreed that their resuscitation roles were clearly stated in the hospital policy.

Exploring the team collaboration during resuscitation indicated that $90.5 \%(\mathrm{n}=162)$ utilized the SBAR mode of communication in resuscitation, and $66.6 \% \quad(n=120)$ underwent post-procedure debriefing.

Only $47.2 \%(n=85)$ agreed with the effectiveness of the other healthcare practitioners roles, and surprisingly, 35\% $(n=63)$ of participants decided to remain neutral. The participants were concerned regarding the other healthcare practitioners' performances in resuscitation, as only $43.9 \%$ $(n=79)$ of nurses were satisfied with the availability of advanced airway practitioners during the code blue.

The study also revealed that $67.2 \%(\mathrm{n}=121)$ of nurses physically replace the medication tray the crash cart. 


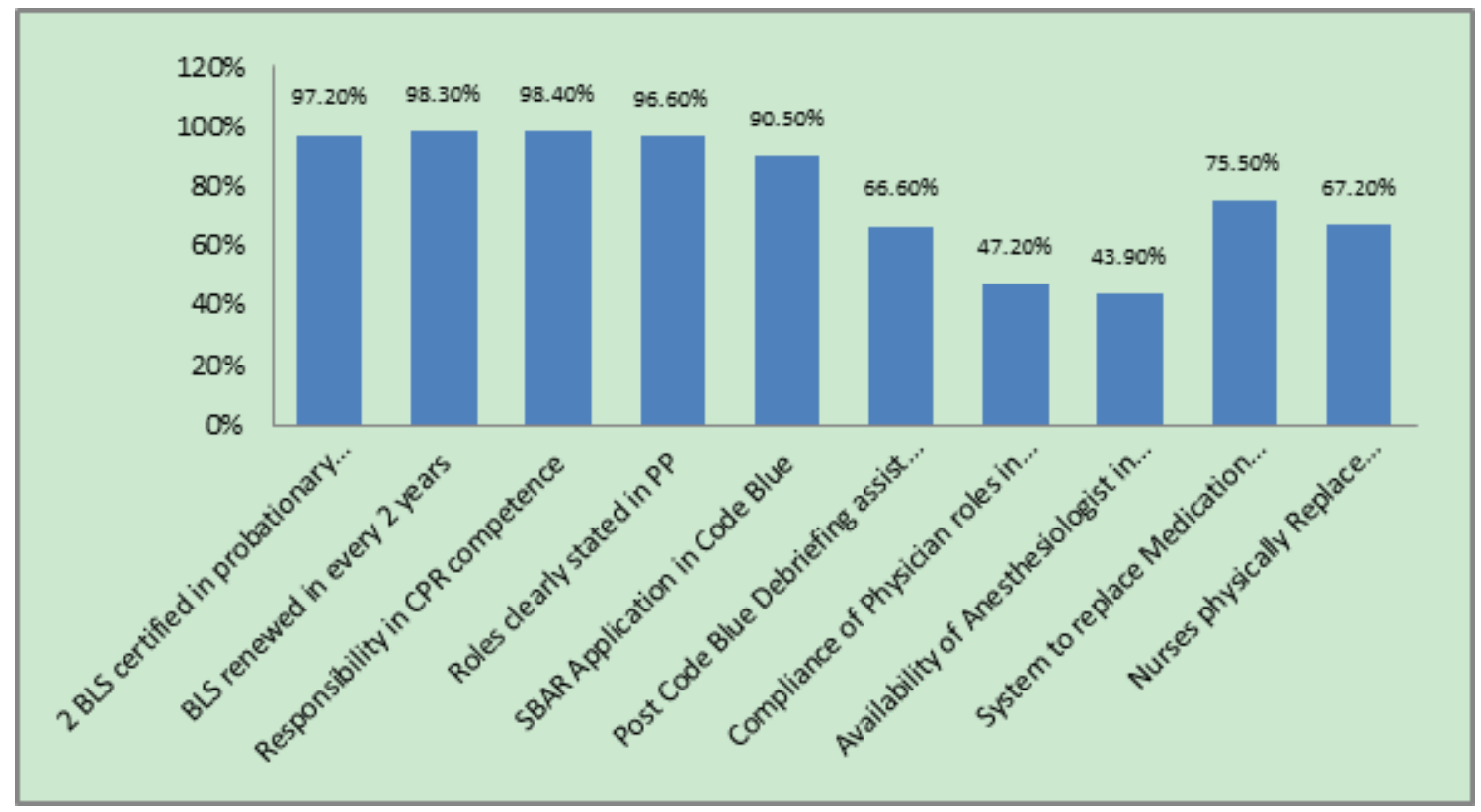

Figure 2. Support and guidance provided for resuscitation care

\section{Finding 3: Standardized and Quality Equipment for Resuscitation Care}

Answering objective 3 in the study on the standardized and quality equipment for resuscitation care, the nurses responded that there are different types of crash carts and defibrillators in the clinical areas. There are three drawer and four drawer crash carts and multiple types of defibrillators used in the hospital, such as Biphasic and Monophasic Physio Control and Nihon Kohden.

Nurses remarked that the quality of ambu bags used during CPR ranged from excellent to poor quality; the mean was 2.54 , with 92 participants agreeing that the quality of the ambu bag after sterilization was in very good to excellent condition (Table 3). The researchers also established that $73.9 \%(n=133)$ of crash carts are not equipped with stop clocks. The majority $(90.6 \% ; n=163)$ of the nurses confirmed that they check their crash cart regularly as per the hospital standard. Finally the study also revealed that electronic resuscitation documentation is not practiced in the studied hospital.

Table 3. Quality of ambu bag after sterilization

\begin{tabular}{|l|c|c|c|}
\hline & $\mathrm{N}$ & Mean & Std. Deviation \\
\hline Quality ambu bag after Sterilization & 180 & 2.54 & 1.005 \\
\hline
\end{tabular}

\section{Finding 4: Application of American Heart Association's Recommendations in Resuscitation Care}

The study provided important information on the application of AHA recommendations in resuscitation care where the requirements are to attend the annual refresher training program and demonstrated that $89.4 \%(n=161)$ of nurses in the teaching hospital comply with attending one or more trainings twice annually at regular intervals. Although nurses are attending the regular training program, this attendance is not reflected in their familiarity with using crash carts during emergency care. Researchers also investigated the availabilities of AEDs for providing initial BLS care as per AHA guidelines. The results measured that $37.5 \%(n=67)$ of the defibrillators are equipped with AEDs and accessibility of their cables and pads is $50.9 \%(\mathrm{n}=91)$. Only $13.9 \%(n=15)$ of nurses are permitted to perform manual defibrillation procedures, while $77.8 \% \quad(n=140)$ stated that they are not allowed to conduct such procedures per the hospital policy.

\section{Discussion}

The total mean of nine questions to assess the effectiveness of the training was $n=2.16$, in which the survey supported that there is existence of the knowledge and skills among nurses in resuscitation after the formal training and preparations. Resuscitation care has to deliver in accordance with the scientific evidence [1], and there is a requirement that medical and nursing staff who respond with resuscitation care be trained [20]. It is further supported that the teaching hospital has a standard for performing such care for the first responders, and they have professional responsibility and accountability for providing that care in clinical settings. It has been documented in other studies that well-trained hospital staff plays an important role in resuscitation care [5]. As per the American Heart Association, it is also necessary to provide simulation training, as this is one of the cornerstones of education in resuscitation and refresher training conducted at regular intervals. This study found that simulation training is in place in the teaching hospital [2]. 
Nurses in the studied hospital can identify emergency statuses and initiate the skills of Basic Life Support in a CAB sequence without defibrillation. Other studies recommended that first responder can provide effective basic life support interventions of resuscitation in the hospital settings in a CABD sequence [22]. However, the study responders are not able to perform early defibrillation in a timely fashion, considering that this procedure is one of the major factors in the resuscitation outcome [19].

Because the study has shown that only a few defibrillators are equipped with the AED, there is a need to update all defibrillators with a complete set of AED options, cables and pads and to make them easily accessible for resuscitation care [17].

It is important to note that there is a need to support and guide all hospital staff in performing effective resuscitation care. Various studies have recommended appropriate resuscitation preparations in hospital settings [20]. The data showed that the hospital is providing formal training and updated refresher trainings at regular intervals.

One of the factors in effective resuscitation care in the hospital is promoting effective team behavior among team members [21]. Team resuscitation performance in the study reflected that some members do not adhere to the hospital resuscitation roles effectively. Efficiency of the resuscitation care is impaired by ineffective team behavior. It is known that teamwork and readiness of equipment are equally important for providing effective resuscitation care [22]. Therefore, the comparison of support and guidance indicated that there is a deficiency area that needs to be considered for better performance.

The teaching hospital supplied crash carts in all clinical areas. The study has shown that resuscitation equipment is not standardized in the teaching hospital; especially crash cart types and defibrillators. Additionally, the quality of the ambu bags is not good after sterilization, which needs to be considered for improving their effectiveness during emergencies. It is encouraging that nurses are maintaining the available resuscitation equipment and are motivated to maintain it to save lives.

Despite higher training rates and annual refresher programs including crash cart awareness, a portion of nurses are not confident in using their crash cart effectively. Adequate resuscitation officers for the trainings are also available in the hospital. However, this study has identified that there is room for improving the resuscitation education and training.

\section{Recommendations}

Because nurses are the first responders in resuscitation, their training should include safe AED and manual defibrillation procedures and permit them to provide such care so that they can provide effective Basic Life support care before a code blue team arrives on the scene. There is a need to update all defibrillators with an AED option with cables and pads, and those items should be readily available in the resuscitation trolley. Other team members, including physicians, should also be included in the safe AED and manual defibrillation training. Policies permitting nurses and physicians to perform defibrillation procedures after their formal training should be implemented. As per AHA guidelines, resuscitation equipment should be standardized and available for emergency care, which needs to take into serious consideration. In order to avoid confusion in its use during resuscitation, it is important to have standardized resuscitation equipment in all clinical areas. The standard for maintaining the available resuscitation trolley needs to be continued; however, it would improve with supervision.

Deficiencies observed in the resuscitation team can be improved through regular Mock Code Blue drills for the actual resuscitation team members. The organization may need to support training, quality monitoring and quality assurance of other healthcare practitioners' performance, which may assist in creating a strong chain of survival in the resuscitation outcomes. There is a need to build excellent resuscitation officers, as there is room for improving their teaching and training.

\section{Conclusions}

Based on the study's findings, nurses are well-prepared and trained for resuscitation care, and they play a vital role in performance. There is a need to establish formal education and training for other resuscitation team members, especially physicians, to improve their performance in resuscitation. Periodic debriefings from the team leader will improve team spirit and may reduce the possibility of future mistakes.

If the corrective measures are incorporated in improvement plans and actions taken effectively, then the team can demonstrate excellent resuscitation practice in the teaching hospital. Actual resuscitation performance and outcomes can be evaluated through further observational study in comparison to these results.

\section{REFERENCES}

[1] Berg, R. A., Hemphill, R., Abella, B. S., Aufderheide, T. P., Cave, D. M., Hazinski, M. F., \& Swor, R. A. (2010). Part 5: Adult basic life support 2010 American Heart Association guidelines for cardiopulmonary resuscitation and emergency cardiovascular care. Circulation, 122(18 suppl 3), S685-S705.

[2] Field, J. M., Hazinski, M. F., Sayre, M. R., Chameides, L., Schexnayder, S. M., Hemphill, R, \& Hoek, T. L. V. (2010). Part 1: executive summary 2010 American Heart Association guidelines for cardiopulmonary resuscitation and emergency cardiovascular care. Circulation, 122(18 suppl 3), S640-S656.

[3] Neumar, R. W., Otto, C. W., Link, M. S., Kronick, S. L., 
Shuster, M., Callaway, C. W., ... \& Morrison, L. J. (2010). Part 8: Adult advanced cardiovascular life support 2010 American Heart Association guidelines for cardiopulmonary resuscitation and emergency cardiovascular care. Circulation, 122(18 suppl 3), S729-S767.

[4] Carpico, B., \& Jenkins, P. (2011). Resuscitation review to improve nursing performance during cardiac arrest. Journal for Nurses in Professional Development, 27(1), 7-12.

[5] Oermann, M. H., Kardong-Edgren, S., Odom-Maryon, T., Hallmark, B. F., Hurd, D., Rogers, N., \& Smart, D. A. (2011). Deliberate practice of motor skills in nursing education: CPR as exemplar. Nursing education perspectives, 32(5), 311-315.

[6] Koziol-McLain, J., Brand, D., Morgan, D., Leff, M., \& Lowenstein, S. R. (2000). Measuring injury risk factors: question reliability in a statewide sample. Injury prevention, $6(2), 148-150$.

[7] Kim, Y. H., Lee, J. H., Lee, D. W., Cho, K. W., Kang, M. J., Kim, Y. W., ... \& Hwang, S. Y. (2015). Differences in Hands-off Time According to the Position of a Second Rescuer When Switching Compression in Pre-hospital Cardiopulmonary Resuscitation Provided by Two Bystanders: A Randomized, Controlled, Parallel Study. Journal of Korean medical science, 30(9), 1347-1353.

[8] Bobrow, B. J., Vadeboncoeur, T. F., Spaite, D. W., Potts, J., Denninghoff, K., Chikani, V., \& Abella, B. S. (2011). The Effectiveness of Ultra brief and Brief Educational Videos for Training Lay Responders in Hands-Only Cardiopulmonary Resuscitation Implications for the Future of Citizen Cardiopulmonary Resuscitation Training. Circulation: Cardiovascular Quality and Outcomes, 4(2), 220-226.

[9] Taniguchi, T., Sato, K., Fujita, T., Okajima, M., \& Takamura, M. (2012). Attitudes to bystander cardiopulmonary resuscitation in Japan in 2010. Circulation Journal, 76(5), 1130-1135.

[10] Meex, I., De Deyne, C., Dens, J., Scheyltjens, S., Lathouwers, K., Boer, W., ... \& Jans, F. (2013). Feasibility of absolute cerebral tissue oxygen saturation during cardiopulmonary resuscitation. Crit Care, 17(2), R 36.

[11] Green, S. M., Roback, M. G., Kennedy, R. M., \& Krauss, B. (2011). Clinical practice guideline for emergency department ketamine dissociative sedation: 2011 update. Annals of emergency medicine, 57(5), 449-461.

[12] Sutton, R. M., Niles, D., Meaney, P. A., Aplenc, R., French, B., Abella, B. S., \& Nadkarni, V. (2011). Low-dose, high-frequency CPR training improves skill retention of in-hospital pediatric providers. Pediatrics, 128(1), e145-e151.

[13] Sawyer, T., Sierocka-Castaneda, A., Chan, D., Berg, B., Lustik, M., \& Thompson, M. (2012). The effectiveness of video-assisted debriefing versus oral debriefing alone at improving neonatal resuscitation performance: a randomized trial. Simulation in Healthcare, 7(4), 213-221.

[14] Terzi, A. B. (2012). Nurse's Role in the Modern Resuscitation Era. Hospital Chronicles, 7(1), 25-31.

[15] Bukiran, A., Erdur, B., Ozen, M., \& Bozkurt, A. I. (2014). Retention of nurses' knowledge after basic life support and advanced cardiac life support training at immediate, 6-month, and 12-month post-training intervals: a longitudinal study of nurses in Turkey. Journal of Emergency Nursing, 40(2), 146-152.

[16] McReynolds, M. C., Mullan, P. B., Fitzgerald, J. T., Kronick, S., Oh, M., \& Andreatta, P. (2013). On-site Simulation Training Improves Nurses' First-Responder Cardiopulmonary Resuscitation Performance: Traveling Simulation Program. Annals of Behavioral Science and Medical Education, 19(1), 8-13.

[17] Mancini, M. E. (2011). Working together, nurses can make a difference in resuscitation outcomes: an update on the American Heart Association's 2010 guidelines for cardiopulmonary resuscitation and emergency cardiovascular care. Japan Journal of Nursing Science, 8(1), 7-10.

[18] Hunziker, S., Tschan, F., Semmer, N. K., Howell, M. D., \& Marsch, S. (2010). Human factors in resuscitation: Lessons learned from simulator studies. Journal of Emergencies, Trauma and Shock, 3(4), 389.

[19] Paul, F. (2010). An exploration of student nurses' thoughts and experiences of using a video-recording to assess their performance of cardiopulmonary resuscitation (CPR) during a mock objective structured clinical examination (OSCE). Nurse education in practice, 10(5), 285-290.

[20] Wissenberg, M., Lippert, F. K., Folke, F., Weeke, P., Hansen, C. M., Christensen, E. F., \& Torp-Pedersen, C. (2013). Association of national initiatives to improve cardiac arrest management with rates of by stander intervention and patient survival after out-of-hospital cardiac arrest. Jama, 310(13), $1377-1384$

[21] Sankar, J., Vijayakanthi, N., \& Sankar, J. (2011). Effect of a Training Module in Cardiopulmonary Resuscitation on the Knowledge and Skills of Pediatric Nursing Personnel. Indian Journal of Emergency Pediatrics, 3(3).

[22] Andreatta, P., Saxton, E., Thompson, M., \& Annich, G. (2011). Simulation-based mock codes significantly correlate with improved pediatric patient cardiopulmonary arrest survival rates*. Pediatric Critical Care Medicine, 12(1), 33-38.

[23] Lighthall, G. K., Poon, T., \& Harrison, T. K. (2010). Using in situ simulation to improve in-hospital cardiopulmonary resuscitation. Joint Commission Journal on Quality and Patient Safety, 36(5), 209-216 